\title{
Hydrodynamic Model for the Plasma-Gas Flow in a Cutting Torch Nozzle
}

\author{
H. Kelly ${ }^{1 *}$ F. O. Minotti ${ }^{1 *}$, L. Prevosto ${ }^{2}$, and B. Mancinelli ${ }^{2}$ \\ ${ }^{1}$ Instituto de Física del Plasma (CONICET), Departamento de Física, \\ Facultad de Ciencias Exactas y Naturales (UBA) Ciudad Universitaria Pab. I, \\ (1428) Buenos Aires, Argentina \\ ${ }^{2}$ Universidad Tecnológica Nacional, Regional Venado Tuerto, Castelli \\ 501, Venado Tuerto, Pcia. Santa Fe, Argentina
}

Received on 5 December, 2003; revised version received on 3 May, 2004

\begin{abstract}
We present a simple hydrodynamic model to obtain the profiles of the relevant physical quantities along a nozzle of arbitrary cross-section in a cutting torch. The model uses a two-zone approximation (a hot central plasma carrying the discharge current wrapped by a relatively cold gas which thermally isolates the nozzle wall from the plasma). Seeking for a solution with sonic conditions at the nozzle exit, the model allows expressing all the profiles in terms of the externally controlled parameters of the torch (geometry of the torch, discharge current, mass flow of the gas and plenum pressure) and the values of the arc and gas temperatures at the nozzle entrance. These last two values can be estimated simply appealing to energy conservation in the cathode-nozzle region. The model contains additional features compared with previous reported models, while retaining simplicity. The detailed consideration of an arc region coupled to the surrounding gas dynamics allows determining voltage drops and consequent delivered power with less assumptions than those found in other published works, and at the same time reduces the set of parameters needed to determine the solution.
\end{abstract}

\section{Introduction}

In metal cutting plasma process an arc plasma jet is directed on a work piece (the metal to be cut) by generating a transferred arc at atmospheric pressure between a cathode and the work piece acting as the anode. A high-quality cut requires a thin, hot and high-velocity plasma jet. That plasma is created by a narrow constricting nozzle inside the torch, into which the gas-plasma system is injected at a high pressure. The fineness of the nozzle creates a large voltage drop in the plasma along its length, providing intense heating of the plasma-gas particles and associated pressure gradient forces, which accelerate the fluids to large velocities[1].

Usually, the development of a plasma-cutting torch is based on an empirical optimisation of the device. In this respect, plasma modelling is an important tool to confront with experimental measurements and to reduce the amount of experimental work used for design optimisation.

Although plasma arc cutting processes have had a widespread industrial development, only recently their plasma characteristics and properties have received attention. Ramakrishnan et al[2, 3] measured the arc voltage, the cathodenozzle voltage, the gas pressure at the nozzle's entrance (the so-called plenum chamber) and the diameter of the plasma jet emerging from the nozzle as functions of the torch arc current. The authors presented also a simplified two-zone fluid model in which, owing to the strong dependence of the gas conductivity with the gas temperature, all the discharge current is assumed to circulate through a hot central core inside the nozzle (the arc region, $T_{A} \sim 25000 \mathrm{~K}$ ), while the remaining structure consists in a relatively cold neutral gas $\left(T_{G} \sim 1000-6000 \mathrm{~K}\right)$ that thermally insulates the nozzle wall from the arc. Assuming sonic conditions at the nozzle exit and a uniform value for the electric field, integral mass and energy balance equations were stated and solved for the exit pressure, arc radius and nozzle potential. A reasonable good agreement with the experimental results was obtained for $T_{A}=25000 \mathrm{~K}$ and $T_{G}=3000 \mathrm{~K}$. Nemchinsky[4] improved Ramakrishnan's work by presenting a more detailed model to describe the plasma-gas flow in the nozzle. Again, hot plasma surrounded by a cold gas flow was considered, and the axial and radial profiles of the plasma temperature and the axial profiles of the other hydrodynamic quantities were calculated in terms of the plasma and gas temperatures and the plasma radius at the nozzle entrance. In Nemchinsky's calculations the cold gas was assumed to evolve isothermally, and the arc and gas velocities were assumed to be the same.

The most complete theoretical plasma studies in cutting torches were presented by González-Aguilar et al[5] and Freton et al[6]. In Ref.[5], a three-dimensional numerical code was developed, which allowed to make predictions on arc voltage, heat transfer to the work piece and pressure exerted by the plasma jet on the material at the cutting region. The code showed good agreement with the experimental arc voltage. In Ref.[6], a two-dimensional turbulent code

${ }^{*}$ Researcher of the CONICET 
was presented and compared with spectroscopic measurements. It was found that turbulence effects were important, mainly in the outer region of the torch (between the nozzle exit and the work piece).

We present in this work a one-dimensional model for the fluid transport along the nozzle of a cutting torch. This approach is justified because in all practical nozzle geometries the nozzle length is much larger than its radius. It should be borne in mind that the present approach does not replace more complete and detailed results obtained from the quoted numerical codes, but it should be taken as a complementary tool to obtain a zero-order solution for the plasma inside the nozzle.

By employing the two-zone approximation, and seeking for a solution with sonic conditions at the nozzle exit, the model gives the spatial profiles of the hydrodynamic quantities and the plasma voltage along a nozzle of arbitrary cross section in terms of the externally controlled torch parameters (discharge current, the geometry of the nozzle, the pressure value at the plenum chamber, the gas mass flow injected in the torch), and the values of $T_{A}$ and $T_{G}$ at the nozzle entrance. These last two values can be estimated by applying a general energy conservation principle in the cathode-nozzle region. All other quantities at the nozzle entrance are determined by the model, and, besides, it will be shown that their values at the nozzle exit depend only weakly on the $T_{A}$ and $T_{G}$ values. Radiative and conductive heat losses from the arc are taken into account, as well as a partial absorption of these losses in the surrounding gas. Predictions of the model are compared with experimental results taken from Ref.[3] and to some extent, with numerical results presented in Ref.[5].

\section{Equations of the model}

If the flow in the nozzle is considered to be stationary and axially symmetric, the continuity, axial and radial momentum, and energy conservation equations can be written respectively as

$$
\begin{gathered}
\frac{\partial}{\partial z}(\rho u)+\frac{1}{r} \frac{\partial}{\partial r}(r \rho v)=0 \\
\rho u \frac{\partial u}{\partial z}=-\frac{\partial p}{\partial z}, \\
\frac{\partial p}{\partial r}=0 \\
\rho u \frac{\partial h}{\partial z}=u \frac{\partial p}{\partial z}-j \frac{\partial V}{\partial z}+\frac{1}{r} \frac{\partial}{\partial r}\left(r k \frac{\partial T}{\partial r}\right)+\varphi
\end{gathered}
$$

where $z$ is the axial coordinate, $r$ the radial coordinate, $u$ is the axial velocity, $v$ the radial velocity, $\rho$ is the fluid density, $h$ its enthalpy, $T$ its absolute temperature, $j$ is the axial electric current density, $V$ the electrostatic potential, $\varphi$ is the density of radiation power either absorbed or emitted, and $k$ the thermal conductivity. In the last three equations, assuming the main flow to be in the axial direction, the radial velocity has been neglected, as well as the radial component of electric current density. Moreover, viscous effects were neglected and axial derivatives of $T$ were also neglected as compared to radial derivatives in the heat transfer term (it was verified in the solutions obtained below that the heat conduction in the axial direction was negligible compared to the terms retained and that the viscous forces in the core of the flow and in the boundary layer at the wall were small compared to the pressure gradient forces).

Consistent with the above approximations we have the following equation for the electrostatic potential

$$
\frac{\partial V}{\partial z}=-\frac{j}{\sigma}
$$

where $\sigma$ is the electrical conductivity. Note that in this problem the magnetic pressure $p_{M}=\mu_{0} I^{2} / 4 \pi R_{A}^{2}$, where $I$ is the total arc current and $R_{A}$ the arc radius, is negligible compared to the thermal pressure: for the typical values $I=100$ A and $R_{A}=5 \times 10^{-4} \mathrm{~m}$ one obtains $p_{M}=1.3 \mathrm{kPa}$, which is much smaller than the thermal pressure as the arc operates at or above atmospheric pressure.

We further consider two well different regions: an internal region of radius $R_{A}(z)$ where all arc current circulates, characterised by high temperature and high electrical conductivity, denominated the arc region, and an external region between $R_{A}(z)$ and the nozzle radius $R_{N}(z)$, of much lower temperature assumed as an electrically non conducting gas, denoted as the gas region. We will consider in this work only straight nozzles $\left(R_{N}=\right.$ const.). No mixing between the hot arc and the cold gas is assumed, since it has been shown[7] that turbulence (leading to a mixing between arc and gas) develops in the diverging region of the nozzle. A sketch of the flow geometry is shown in Fig. 1. If Eq. (1a) is multiplied by $2 \pi r$ and radially integrated between $r=0$ and $r=R_{A}(z)$ one obtains the mass conservation for the arc region. Analogously, integrating between $r=R_{A}(z)$ and $r=R_{N}(z)$, one obtains the mass conservation for the gas region. The results are

$$
\frac{\partial}{\partial z} \int_{0}^{R_{A}} 2 \pi \rho u r d r=0
$$

$$
\frac{\partial}{\partial z} \int_{R_{A}}^{R_{N}} 2 \pi \rho u r d r=0
$$

where it was used that $v\left(r=R_{A}\right)=u\left(r=R_{A}\right) d R_{A} / d z$. 


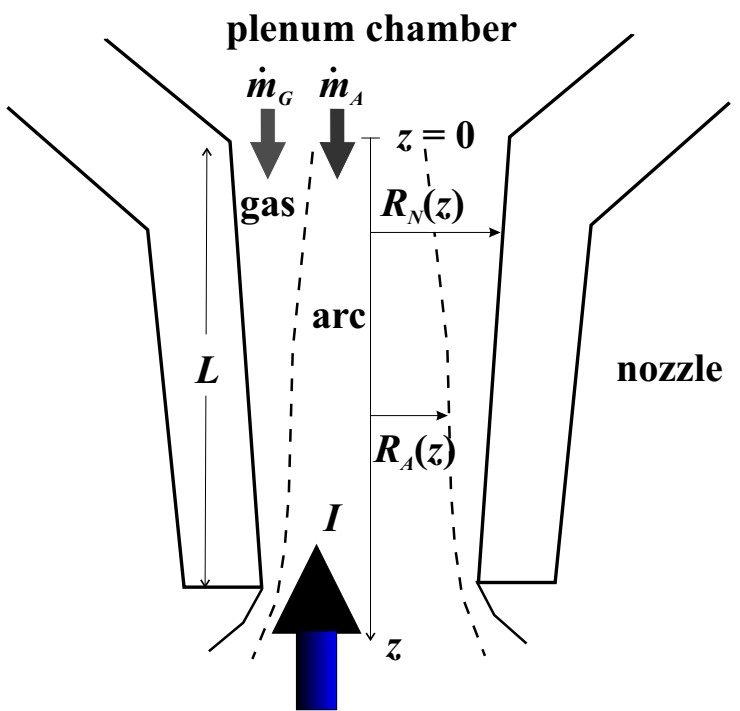

Figure 1. Sketch of the flow geometry in the nozzle.

We further model each region as having uniform characteristics in the radial direction, so that, for instance, we define a uniform arc temperature $T_{A}(z)$ for $0 \leq r \leq R_{A}(z)$ and a uniform gas temperature $T_{G}(z)$ for $R_{A}(z) \leq r \leq$ $R_{N}(z)$. Using these constant by pieces radial profiles Eqs. (1b) and (1d) are multiplied by $2 \pi r$, integrated between $r=0$ and $r=R_{A}(z)$, and then divided by $\pi R_{A}^{2}$ to obtain the equations for the arc region, while if integrated between $r=R_{A}(z)$ and $r=R_{n}(z)$ and then divided by $\pi\left(R_{N}^{2}-R_{A}^{2}\right)$ one gets the equations for the gas region. The final system is then obtained including the integrated version of Eqs. (3a) and (3b), and given by

$$
\begin{gathered}
\rho_{A} u_{A} \pi R_{A}^{2}=\dot{m}_{A}, \\
\rho_{A} u_{A} \frac{d u_{A}}{d z}=-\frac{d p}{d z} \\
\rho_{A} u_{A} C_{p}^{A} \frac{d T_{A}}{d z}=u_{A} \frac{d p}{d z}+\frac{1}{\sigma}\left(\frac{I}{\pi R_{A}^{2}}\right)^{2}-\chi-\varphi_{A}, \\
\rho_{G} u_{G} \pi\left(R_{N}^{2}-R_{A}^{2}\right)=\dot{m}_{G}, \\
\rho_{G} u_{G} \frac{d u_{G}}{d z}=-\frac{d p}{d z}, \\
\rho_{G} u_{G} C_{p}^{G} \frac{d T_{G}}{d z}=u_{G} \frac{d p}{d z}+\frac{R_{N}^{2}-R_{A}^{2}}{R_{A}^{2}} \chi+\varphi_{G}, \\
p_{A}=p_{G},
\end{gathered}
$$

where the last equation expresses the uniformity of pressure in the radial direction, $\dot{m}_{A}$ and $\dot{m}_{G}\left(\dot{m}_{A}+\dot{m}_{G}=\dot{m}=\right.$ const.) are the mass flow rates in the arc and gas regions, respectively, $\chi$ is the rate of energy density lost by the arc region by thermal conduction, $\varphi_{A}$ is the density of radiation power emitted from the arc, $\varphi_{G}$ that absorbed by the gas, and $C_{p}^{A}$ and $C_{p}^{G}$ are the specific thermal capacities at constant pressure for the arc and gas, respectively, defined as $\left.C_{p}=\partial h / \partial T\right)_{p}$. The heat loss from the gas to the nozzle wall was neglected as compared to the heat received from the arc region, and Eq. (2) was used in Eq. (4c) with $j=I /\left(\pi R_{A}^{2}\right)$ to rewrite the Joule heating. The expression of $\chi$ and the approximation used to model it are

$$
\left.\chi \equiv-\frac{2 k_{G} R_{A}}{R_{N}^{2}-R_{A}^{2}} \frac{\partial T}{\partial r}\right)_{R_{A}} \simeq \frac{2 \lambda k_{G} R_{A}\left(T_{A}-T_{G}\right)}{\left(R_{N}+R_{A}\right)\left(R_{N}-R_{A}\right)^{2}},
$$

where $k_{G}$ is the thermal conductivity of the gas and $\lambda$ is a numerical constant of order unity used as a parameter of the model. Since $C_{p}^{A}, C_{p}^{G}, k_{G}, \sigma, \varphi_{A}$, and $\varphi_{G}$ are given as functions of the thermodynamic variables of the corresponding regions, the system (4) is closed once the equation of state is given for each region. A given solution is obtained if the mass rates $\dot{m}_{A}$ and $\dot{m}_{G}$, and the geometry of the nozzle $R_{N}(z)$ are specified, and the values of velocities, densities and temperatures, compatible with Eq. (4g), given at some axial position.

As with the much simpler problem of adiabatic flow in a nozzle, the system (4a)-(4g) has sonic points where some of the equations become singular. To study this problem it is convenient to consider the generic equations for either the arc (Eqs. (4a)-(4c)) or the gas region (Eqs. (4d)-(4f)) written as:

$$
\begin{gathered}
\frac{1}{\rho} \frac{d \rho}{d z}+\frac{1}{u} \frac{d u}{d z}+\frac{1}{S} \frac{d S}{d z}=0, \\
\rho u \frac{d u}{d z}=-\frac{d p}{d z} \\
\rho u C_{p} \frac{d T}{d z}=u \frac{d p}{d z}+W
\end{gathered}
$$

where Eq. (6a) is the derivative of the logarithm of either Eq. (4a) or Eq. (4d), $S$ is the cross section of the given region (arc or gas) and $W$ represents the rate of change of specific heat (the sum of Joule heating, heat loss by conduction, and radiated power for the arc region, and heat gained by conduction plus absorbed radiation power for the gas region). Given the equation of state, the pressure derivatives are written as

$$
\left.\left.\frac{d p}{d z}=\frac{\partial p}{\partial T}\right)_{\rho} \frac{d T}{d z}+\frac{\partial p}{\partial \rho}\right)_{T} \frac{d \rho}{d z}
$$

and so Eqs. (6a)-(6c) represent a linear system for the derivatives of $u, T$ and $\rho$. The condition of zero determinant of the matrix of coefficients of this system determines the singular point:

$$
\text { Det } \left.\left.=u^{2}\left[\rho C_{p}-\frac{\partial p}{\partial T}\right)_{\rho}\right]-\rho C_{p} \frac{\partial p}{\partial \rho}\right)_{T}=0,
$$

which corresponds to the point where the velocity takes on the value of the sound velocity defined by

$$
c_{S}^{2}=\frac{\left.\frac{\partial p}{\partial \rho}\right)_{T}}{\left.1-\frac{1}{\rho C_{p}} \frac{\partial p}{\partial T}\right)_{\rho}} .
$$

All physical magnitudes must have finite derivatives at this sonic point, so that, solving for the derivatives and imposing that for each of them the factor that multiplies $D^{-1} t^{-1}$ 
must be equal to zero at the sonic point, one obtains the unique condition for this to happen

$$
\frac{1}{S} \frac{d S}{d z}=\frac{\left.\left.W \frac{\partial p}{\partial T}\right)_{\rho}\left[\rho C_{p}-\frac{\partial p}{\partial T}\right)_{\rho}\right]^{1 / 2}}{\left.\rho^{5 / 2} C_{p}^{3 / 2}\left[\frac{\partial p}{\partial \rho}\right)_{T}\right]^{3 / 2}} .
$$

In this way, if the flow of either the arc or the gas region reaches the sonic velocity at a given axial position, the derivative of the section of the region at that point must be given by Eq. (10) for the solution to be physically acceptable.

To solve the system (4a)-( $4 \mathrm{~g})$, equations of state for the arc and gas must be supplied. Ideal gas expressions were employed of the form:

$$
\begin{gathered}
p_{A}=(1+\alpha) \rho_{A} \mathcal{R}_{A} T_{A}, \\
p_{G}=\rho_{G} \mathcal{R}_{G} T_{G},
\end{gathered}
$$

where $\alpha$ is the degree of ionization in the arc and it was assumed that $T_{G}$ is low enough so that ionization or dissociation (in the case of a molecular gas) are negligible in this region. $\alpha$ was evaluated from Saha equation[8] assuming LTE equilibrium. Other coefficients of the problem like $C_{p}^{A}, C_{p}^{G}, k_{A}$, $k_{G}$ and $\sigma$ were obtained from Ref.[9] for the temperature range $300-45000 \mathrm{~K}$. Radiative losses from the arc were included by considering that the total radiation field is composed by $\sim 50 \%$ of continuous radiation and another $50 \%$ of line radiation[10,11]. The surrounding gas is considered to be transparent to continuous radiation but opaque to line radiation.

\section{Results}

The radiation term in Eq. (6c) is always positive in our case because, for the arc, the radiated power plus the conductive heat loss is a fraction of the Joule heating, while the gas always receives heat from the arc. Eq. (10) then imposes that the cross section of the region that reaches sonic velocity must be increasing at that point. In the case of a straight nozzle if the cross section increases for one region it decreases for the other, so that the gas and the arc flow cannot reach the sonic velocity simultaneously. It is well known[12] from the theory of single flows in finite nozzles of constant cross section that if the flow is always heated $(W>0$ at all points) it is either subsonic at all points or becomes sonic only at the nozzle exit. If more heat is added to a flow that was sonic at the nozzle exit, the conditions at the nozzle entry adjust so that again the flow becomes sonic at the exit. We will follow the guidelines given by this simpler quoted case and consider that the strongly heated arc flow becomes sonic at the nozzle exit. This condition is commonly used in torch models and is well verified in numerical simulations [5, 6].

The actual solution of the system $(4 \mathrm{a})-(4 \mathrm{~g})$ then proceeds as follows: the mass flow $\dot{m}$ in the nozzle is a given parameter as well as the plenum pressure $p_{0}$ at which this flow is injected in the nozzle entry. If only the temperatures $T_{A}$ and $T_{G}$ are fixed at the nozzle entry, the condition on the pressures $p_{A}=p_{G}=p_{0}$ also determines the densities $\rho_{A}$ and $\rho_{G}$ at that point. On the other hand one has $\dot{m}=\dot{m}_{A}+\dot{m}_{G}$, so that once a value of, say, $\dot{m}_{G}$ and a value of $R_{A}$ are chosen at the nozzle entry, the velocities $u_{A}$ and $u_{G}$ are determined at that point by Eqs. (4a) and (4d), and the system (4a)-(4g) can be integrated. There are however unique values of $\dot{m}_{G}$ and of $R_{A}$ that ensure that either $u_{A}$ or $u_{G}$ (whichever does it first) becomes sonic at the nozzle exit and simultaneously condition (10) is satisfied at the same point. In this way, given the values of both temperatures at the nozzle entry there is only a physically acceptable solution for given $\dot{m}, p_{0}$ and nozzle dimensions.

In the Figs. 2 spatial profiles obtained from the model are presented for a nozzle with $I=100 \mathrm{~A}, R_{N}=0.75$ $\mathrm{mm}, L=3.9 \mathrm{~mm}$, operated with $N_{2}$ gas, $p_{0}=0.45 \mathrm{MPa}$, and with $\dot{m}=0.42 \mathrm{~g} / \mathrm{s}$. These parameters correspond to one of the experimental cases presented by Ramakrishnan et al[3]. The numerical parameter that regulates the arcgas heat exchange by thermal conduction (Eq. (8)) was taken $\lambda=1$. In Fig. 2a) the arc radius $R_{A}(z)$ is plotted for $T_{G}(z=0)=1000 \mathrm{~K}$ and three different values of $T_{A}(z=0)=10000,15000$ and 20000 K. The other Figs. 2 show $p(z)$ (Fig. 2b)), $T_{A}(z)$ (Fig. 2c)), $T_{G}(z)$ (Fig. 2d)), $u_{A}(z)$ (Fig. 2e)), and $u_{G}(z)$ (Fig. 2f)), arc Mach number $M_{A}$ (Fig. 2g)), and gas Mach number $M_{G}$ (Fig. 2h)) for the same entrance temperatures. The values of $\dot{m}_{G} / \dot{m}$ and of $R_{A} / R_{N}$ for the three considered cases resulted to be $\dot{m}_{G} / \dot{m}=0.926,0.923$ and 0.919 , and $R_{A} / R_{N}=0.324$, 0.436 and 0.693 , respectively, and the corresponding nozzle voltage drops $(\Delta V \equiv V(z=L)-V(z=0))$ were $\Delta V$ $=69.8,60$ and $44.4 \mathrm{~V}$, respectively. The total electrical power delivered into the nozzle $(6.98,6$ and $4.44 \mathrm{~kW}$, respectively) almost coincides with the power carried by the arc and gas (enthalpy plus kinetic energy fluxes), indicating that radiative losses are not very relevant. The power delivered into the arc is typically 10 times larger than that carried by the gas, and the enthalpy flux largely exceeds the corresponding kinetic energy flux, for both the arc and gas systems. It can be seen from Fig. 2a) that the arc radius expands along the nozzle for $T_{A}(z=0)=10000$ or $15000 \mathrm{~K}$, but slightly contracts for the highest temperature considered. However, the final arc radius at the nozzle exit, $R_{A}(z=L) \simeq 0.47-0.48 \mathrm{~mm}$, results almost independent of $T_{A}(z=0)$, (the numerical differences in the values of $R_{A}(z=L)$ are indistinguishable from an experimental point of view) and its value is close to that measured in Ref.[3]. Fig. 2b) shows a decreasing profile of $p(z)$, which again results only slightly dependent on $T_{A}(z=0)$. The total pressure drop in the nozzle is approximately $0.2 \mathrm{MPa}$. From Fig. 2c) it can be seen that $T_{A}(z)$ monotonically increases along the nozzle, but reaches a final value again almost independent of its entrance value. The gas temperature also increases along the nozzle, with a profile only slightly depending on $T_{A}(z=0)$. From Fig. 2e) and 2f) it can be seen that the arc and gas velocities continuously increases with $z$, and show also very weak dependence on $T_{A}(z=0)$. In Figs. 2.g) and 2.h) the Mach numbers are seen to increase continuously; in all the cases considered the arc reaches sonic conditions at the nozzle exit, which is not clearly appreciated in the figures as the final increase is very steep and is not shown. 

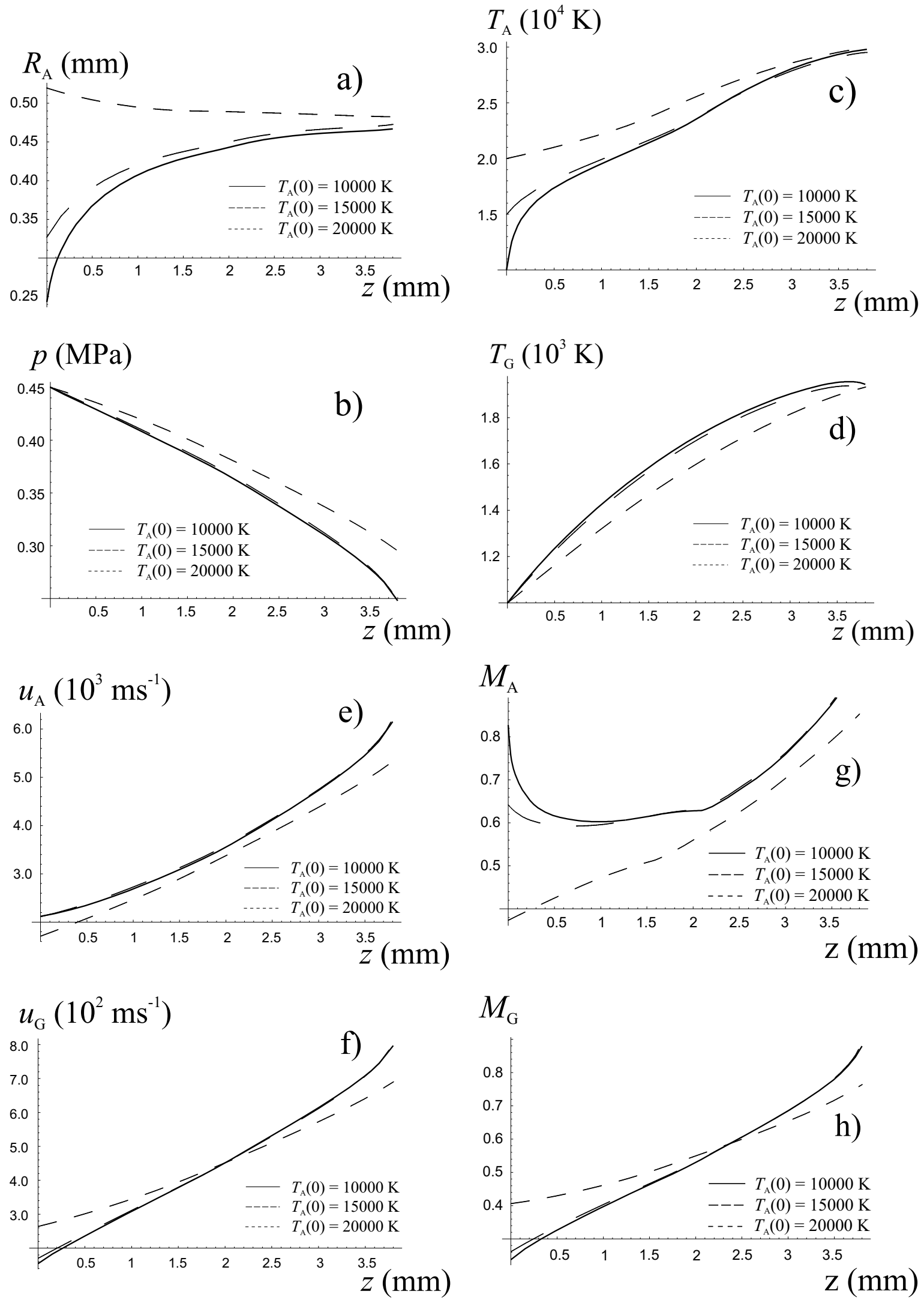

Figure 2. Spatial profiles obtained from the model for a nozzle with $I=100 \mathrm{~A}, R_{N}=0.75 \mathrm{~mm}, L=3.9 \mathrm{~mm}$, operated with $N_{2}$ gas, $p_{0}=0.45 \mathrm{MPa}$, and with $\dot{m}=0.4 \mathrm{~g} / \mathrm{s}(\lambda=1)$. The quantities are plotted for $T_{G}(z=0)=1000 \mathrm{~K}$ and three different values of $T_{A}(z=0)=10000,15000$ and 20000 K. Fig. 2a): arc radius; Fig. 2b): pressure; Fig. 2c): arc temperature; Fig. 2d): gas temperature; Fig. 2e): arc velocity; Fig. 2f): gas velocity, Fig. 2g): arc Mach number, and Fig. 2h): gas Mach number. 
TABLE I. Physical quantities at the nozzle entrance and exit for $R_{N}=0.75 \mathrm{~mm}, L=3.9 \mathrm{~mm}$.

\begin{tabular}{|l|l|l|l|l|l|l|l|l|l|l|l|l|l|}
\hline $\begin{array}{l}I \\
(\mathrm{~A})\end{array}$ & $\begin{array}{l}p_{o} \\
(\mathrm{MPa})\end{array}$ & $\begin{array}{l}\dot{m} \\
(\mathrm{~g} / \mathrm{s})\end{array}$ & $\begin{array}{l}T_{A} \\
(z=0) \\
\left({ }^{\circ} \mathrm{K}\right)\end{array}$ & $\begin{array}{l}T_{G} \\
(z=0) \\
\left({ }^{\circ} \mathrm{K}\right)\end{array}$ & $\dot{m}_{G} / \dot{m}$ & $\begin{array}{l}R_{A} \\
(z=0) \\
(\mathrm{mm})\end{array}$ & $\begin{array}{l}R_{A} \\
(z=L) \\
(\mathrm{mm})\end{array}$ & $\begin{array}{l}T_{A} \\
(z=L) \\
\left({ }^{\circ} \mathrm{K}\right)\end{array}$ & $\begin{array}{l}T_{G} \\
(z=L) \\
\left({ }^{\circ} \mathrm{K}\right)\end{array}$ & $\begin{array}{l}u_{A} \\
(z=L) \\
(\mathrm{m} / \mathrm{s})\end{array}$ & $\begin{array}{l}u_{G} \\
(z=L) \\
(\mathrm{m} / \mathrm{s})\end{array}$ & $\begin{array}{l}P \\
(z=L) \\
(\mathrm{MPa})\end{array}$ & $\begin{array}{l}\Delta V \\
(\mathrm{~V})\end{array}$ \\
\hline 40 & 0.31 & 0.42 & 10000 & 1000 & 0.99 & 0.17 & 0.27 & 35400 & 1600 & 6700 & 760 & 0.17 & 56 \\
\hline & & & 15000 & 1000 & 0.98 & 0.23 & 0.32 & 30900 & 1500 & 6300 & 740 & 0.17 & 49 \\
\hline & & & 20000 & 1000 & 0.98 & 0.36 & 0.33 & 30500 & 1500 & 6300 & 750 & 0.18 & 35 \\
\hline & & & 10000 & 2000 & 0.97 & 0.46 & 0.34 & 19700 & 2500 & 3700 & 1040 & 0.21 & 43 \\
\hline & & & 15000 & 2000 & 0.97 & 0.46 & 0.35 & 19800 & 2500 & 3800 & 1030 & 0.20 & 35 \\
\hline & & & 20000 & 2000 & 0.97 & 0.46 & 0.34 & 27100 & 2500 & 4800 & 1040 & 0.21 & 28 \\
\hline 100 & 0.45 & 0.4 & 10000 & 1000 & 0.93 & 0.24 & 0.47 & 29800 & 1900 & 6200 & 810 & 0.24 & 70 \\
\hline & & & 15000 & 1000 & 0.92 & 0.33 & 0.47 & 29500 & 1900 & 6300 & 810 & 0.25 & 60 \\
\hline & & & 20000 & 1000 & 0.92 & 0.52 & 0.48 & 30100 & 1900 & 6300 & 810 & 0.25 & 44 \\
\hline & & & 10000 & 2000 & 0.88 & 0.58 & 0.51 & 18600 & 2700 & 3800 & 1100 & 0.28 & 55 \\
\hline & & & 15000 & 2000 & 0.88 & 0.59 & 0.52 & 19800 & 2700 & 4100 & 1100 & 0.29 & 47 \\
\hline & & & 20000 & 2000 & 0.90 & 0.58 & 0.58 & 26800 & 2800 & 5400 & 1100 & 0.28 & 37 \\
\hline 160 & 0.54 & 0.35 & 10000 & 1000 & 0.87 & 0.30 & 0.54 & 31300 & 2400 & 6300 & 880 & 0.29 & 75 \\
\hline & & & 15000 & 1000 & 0.87 & 0.39 & 0.54 & 31200 & 2400 & 6300 & 870 & 0.29 & 66 \\
\hline & & & 20000 & 1000 & 0.85 & 0.58 & 0.56 & 31000 & 2200 & 6300 & 830 & 0.31 & 49 \\
\hline & & & 10000 & 2000 & 0.86 & 0.60 & 0.55 & 26900 & 3100 & 5400 & 1140 & 0.30 & 67 \\
\hline & & & 15000 & 2000 & 0.85 & 0.62 & 0.56 & 26900 & 3100 & 5400 & 1140 & 0.31 & 59 \\
\hline & & & 20000 & 2000 & 0.83 & 0.64 & 0.58 & 28800 & 3000 & 5600 & 1140 & 0.33 & 45 \\
\hline
\end{tabular}

To illustrate the results of the model for different input parameters, in Table I are presented several quantities at the nozzle entrance and exit for the quoted nozzle geometry and for $T_{A}(z=0)=10000,15000$ and $20000 \mathrm{~K}$, $T_{G}(z=0)=1000$ and $2000 \mathrm{~K}$ and $I=40,100$ and 160 A. The values of the discharge current correspond to cases in which the arc radius at the nozzle exit was experimentally determined[3]. It can be seen from the Table that for a given value of $T_{A}(z=0)$, the values of the arc and gas temperatures and velocities at the nozzle exit are only weakly dependent on $I$. The most important changes in the quantities appear at the nozzle entrance, where an increase of $I$ is associated with a thicker arc carrying a higher mass at $z=0$. The total pressure and voltage drops along the nozzle both increase as $I$ increases. An increase in the $T_{G}(z=0)$ value produces also thicker arcs with higher masses at the nozzle entrance, and a colder and slower plasma but a hotter and faster gas at the nozzle exit. The pressure and voltage drops along the nozzle both decrease in this case. The arc in the nozzle can be expanding (for small $T_{A}(z=0)$ and $T_{G}(z=0)$ values), or contracting in the opposite case.

To investigate the influence of the heat power exchanged between the arc and gas by thermal conduction, the parameter $\lambda$ that regulates this process was varied between 0.2 and 5 (that is, a 25 -fold total variation). It was found that the quantities at the nozzle exit depended very weakly on $\lambda$, but again the values of the quantities at the nozzle entrance changed strongly with $\lambda$. A large $\lambda$ value produces a thick and slow arc, and, correspondingly, a thin and fast gas at the nozzle entrance. However the quantity $\dot{m}_{G} / \dot{m}$ remains practically unaltered.

It is worth comparing the numerical results with experimental data reported in[3] with a similar torch. The experimental arc radius reported in[3] were: for $I=40 \mathrm{~A}$, $R_{A}(z=L)=0.33 \mathrm{~mm}$; for $I=100 \mathrm{~A}, R_{A}(z=L)=$ $0.51 \mathrm{~mm}$; and for $I=160 \mathrm{~A}, R_{A}(z=L)=0.57 \mathrm{~mm}$.
These values are close to the values shown in Table I for $T_{G}(z=0)=1000 \mathrm{~K}$ and for the highest $T_{A}(z=0)$ values. Other values of $R_{A}(z=L)$ reported in[3] for different $I$ values are also rather well reproduced in our calculations.

In practice, the values of $T_{A}(z=0)$ and $T_{G}(z=0)$ can be somewhat determined by applying a general energy conservation principle in the cathode-nozzle region. The power supplied into the arc in this region can be estimated as $W_{c n} \simeq I \Delta V_{c n} \simeq I^{2} L_{c n} / \sigma \pi R_{A}^{2}(z=0)$, where $L_{c n}$ is the distance between the cathode surface and the nozzle entrance. In turn, $W_{c n}$ should be equal to the power carried by the arc-gas system at the nozzle entrance $\left(W^{\prime}\right)$, given as: $W^{\prime}=\dot{m}_{A}\left(u_{A}^{2} / 2+h_{A}\right)+\dot{m}_{G}\left(u_{G}^{2} / 2+h_{G}\right)$ (the initial enthalpy and kinetic energy of the gas can be neglected). For instance, taking again the case with $I=100$ A with the torch geometry published in[3] $\left(L_{c n}=2 \mathrm{~mm}\right)$, $W_{c n} \simeq W^{\prime} \simeq 4 \mathrm{~kW}$ for $T_{A}(z=0)=18000 \mathrm{~K}$, and $T_{G}(z=0)=1100 \mathrm{~K}$. With these values, the quantities at the nozzle exit take the following values: $T_{A}(z=L)=$ $29700 \mathrm{~K}, T_{G}(z=L)=1900 \mathrm{~K}, u_{A}(z=L)=6300$ $\mathrm{m} / \mathrm{s}, u_{G}(z=L)=800 \mathrm{~m} / \mathrm{s}, p(z=L)=0.25 \mathrm{MPa}$, $\Delta V=50 \mathrm{~V}$. It must be noted that the sensitivity of the equation $W_{c n} \simeq W^{\prime}$ to changes of $T_{A}(z=0)$ is much larger than that corresponding to changes of $T_{G}(z=0)$, due to the fact that the power carried by the plasma is much larger than that carried by the gas. Hence, this equation is employed basically to specify $T_{A}(z=0)$. Given the low sensitivity of the exit values of the quantities to $T_{G}(z=0)$, it is not necessary to determine this value very accurately.

\section{Discussion and final remarks}

We have developed a simple and low computing time model which allows to obtain the profiles of the relevant physical quantities along a nozzle in a cutting torch, in terms of the 
externally-controlled parameters of the torch (geometry of the torch, discharge current, mass flow of the gas and plenum pressure) and the values of the arc and gas temperatures at the nozzle entrance. From a general energy conservation principle applied to the cathode-nozzle region, these last values (mainly the arc temperature) are in turn relatively fixed by the external torch parameters. Although the results presented in this work are restricted to straight nozzles, it is worth noting that the model is valid for nozzles of arbitrary cross-section.

The model contains additional features compared with previous reported models, while retaining simplicity. The detailed consideration of an arc region coupled to the surrounding gas dynamics allows determining voltage drops and consequent delivered power with less assumptions than those in Refs.[2, 3], and at the same time reduces the set of parameters needed to determine the solution. Compared with the model in Ref.[4], it removes the conditions of isothermal evolution of the gas and of equal velocities of arc and gas flows, which are not well verified in the simulations in of Refs.[5, 6] as deduced from the temperature and Mach number profiles in the nozzle region. Moreover, Steenbeck's principle is not required to determine the initial arc radius, as was done in Ref.[4].

Comparison with the much more complete existing codes is not easy as much freedom exists in the determination of arc and gas regions and their averaged magnitudes. An exception is the pressure, which is rather uniform in the nozzle section for each axial position, and at the same time its change along the nozzle is very sensitive to different arc parameters. We have verified that the pressure drops in the nozzle presented in Ref.[5] are well reproduced by our onedimensional model. For instance, in the case with $I=50$ A and mass flow of $20 \mathrm{lpm}$, according to our model the pressure drops from $2 \mathrm{~atm}$ at the nozzle entrance to $1.3 \mathrm{~atm}$ at the exit, while for a mass flow of $40 \mathrm{lpm}$ the drop is from $3.2 \mathrm{~atm}$ to $2.1 \mathrm{~atm}$. These values are in good agreement with the reported pressure changes between plenum chamber and pressure at the sonic point (in the simulations the sonic point is sometimes reached inside the nozzle, very close to the exit; and a significant, up to $0.4 \mathrm{~atm}$, additional pressure drop is verified between this point and the actual exit).

A particularly convenient feature of the model is that given the values of relatively simple to measure quantities as gas flow and plenum pressure, together with nozzle dimen- sions, the assumption of sonic condition at the nozzle exit reduces the required parameters to only two temperatures, which in turn can be simply estimated appealing to energy conservation in the cathode-nozzle region. In this sense the model is predictive, which together with its simplicity, makes it valuable for design purposes.

\section{Acknowledgments}

This work was supported by a grant from the Buenos Aires University.

\section{References}

[1] R. C. Fernicola, Weld. J. 77, 52 (1998).

[2] S. Ramakrishnan, M. Gershenzon, F. Polivka, T. N. Kearny, and M. W. Rogozinsky, IEEE Trans. Plasma Sci. 25, 937 (1997).

[3] S. Ramakrishnan and M. W. Rogozinsky, J. Phys. D: Appl. Phys. 30, 636 (1997).

[4] V. A. Nemchinsky, J. Phys. D: Appl. Phys. 31, 3102 (1998).

[5] J. González-Aguilar, C. Pardo Sanjurjo, A. Rodríguez-Yunta, and M. A. García Calderón, IEEE Trans. Plasma Sci. 27, 264 (1999).

[6] P. Freton, J. J. Gonzalez, A. Gleizes, F. Camy Peyret, G. Caillibotte, and M. Delzenne, J. Phys. D: Appl. Phys. 35, 115 (2002).

[7] W. Hermann, U. Kogelschatz, 1. Niemeyer, K. Ragaller, and E. Shade, J.Phys. D: Appl. Phys. 7, 1703 (1974).

[8] Y. P. Raizer Gas Discharge Physics Springer-Verlag (1991).

[9] G. Speckhofer, R. Gilles, W. Smith, and M. Bures, A consistent set of thermodynamic properties and transport coefficients for high temperature plasmas Proc. 14th Int. Symp. On Plasma Chemistry M. Hrabosky, M. Konrad and V. Kopecky eds. Vol. 1, 269-74, Inst. Plasma Physics, Prague, Czech Republic (1999).

[10] D. L. Evans and R. S. Tankin, Phys. Fluids 10, 1137 (1967).

[11] R. U. Krey and J. C. Morris, Phys. Fluids 13, 1483 (1970).

[12] L. D. Landau and E. M. Lifshitz, Fluid Mechanics (Course of Theoretical Physics, Vol. 6), Butterworth-Heinemann Ltd, Oxford (1987). 